\title{
Propofol administration to the fetal-maternal unit reduces cardiac oxidative stress in preterm lambs subjected to prenatal asphyxia and cardiac arrest
}

\author{
Matthias Seehase ${ }^{1,2}$, Patrick Houthuizen ${ }^{3}$, Jennifer J.P. Collins ${ }^{1,4}$, Luc J. Zimmermann', Boris W. Kramer ${ }^{1}$
}

BACKGROUND: Little is known about the effects of propofol on oxidative stress and its effect on key structures of the contractile apparatus as the myosin light chain 2 (MLC2) and the p38MAPK survival pathway in the preterm heart. We hypothesized that propofol administration could attenuate the hypoxic myocardial injury after birth asphyxia.

METHODS: Pregnant ewes were randomized to receive either propofol or isoflurane anesthesia. A total of 44 late-preterm lambs were subjected to in utero umbilical cord occlusion (UCO), resulting in asphyxia and cardiac arrest, or sham treatment. After emergency cesarean delivery, each fetus was resuscitated, mechanically ventilated, and supported under anesthesia for $8 \mathrm{~h}$ using the same anesthetic as the one received by its mother.

RESULTS: At $8 \mathrm{~h}$ after UCO, occurrence of reactive oxygen species and activation of inducible nitric oxide synthase in the heart were lower in association with propofol anesthesia than with isoflurane. This was accompanied by less degradation of MLC2 but higher p38MAPK level and in echocardiography with a trend toward a higher median left ventricular fractional shortening.

CONCLUSION: The use of propofol resulted in less oxidative stress and was associated with less cytoskeletal damage of the contractile apparatus than the use of isoflurane anesthesia.

P renatal asphyxia can affect all organs due to poor perfusion and reduced oxygen delivery, but the most detrimental effects are observed in the central nervous and cardiovascular system $(1-5)$. Therefore, it is not surprising that asphyxia is the third major cause of neonatal mortality and accounts for more than one million neonatal deaths worldwide per year (6). Although resuscitation revives about three-quarters of these newborns (7), late complications of asphyxia still remain an important cause of morbidity and mortality in children (6,7). Cardiovascular dysfunction is the most common problem which occurs in more than $60 \%$ of asphyxiated neonates $(2,8)$. Therefore, it is crucial to elucidate and to understand the course of hypoxic injury leading to cardiac dysfunction. This knowledge will give us the option to develop therapeutic strategies which prevent or reverse the detrimental outcomes which we see in our neonatal units.

Our previous research found that the use of propofol for maternal anesthesia resulted in less fetal cardiac injury in late-preterm lambs subjected to severe asphyxia than the use of isoflurane anesthesia (4). We could show that this cardioprotective effect was mediated partly by interaction with the mitochondrial apoptotic pathway. However, our previous data indicated that the reduction in cardiac injury did not result exclusively from the interaction of propofol with the mitochondrial apoptotic pathway. Therefore, the cellular therapeutic mechanism of propofol in birth asphyxia is not completely understood. Recent evidence has shown that asphyxia affects key structures of the contractile apparatus. One such structure is the myosin light chain (MLC) 2 which is a crucial component for the ability of the heart muscle to contract and, correspondingly, to beat. But under hypoxic conditions, MLC2 is assaulted by reactive oxygen species (ROS), especially in the presence of nitric oxide (NO), by the aggressive fusion product peroxynitrite. This results in MLC2 degradation and subsequent systolic dysfunction (9). However, previous research has indicated that propofol might interfere with this cascade (10).

Propofol operates via many mechanisms that may influence the degree of ischemia and subsequent reperfusion injury. It influences crucial components of the immune system such as nuclear factor 'kappa-light-chain-enhancer' of activated B cells (11) or members of the signal transducer and activator of transcription family $(4,5)$, help to maintain cellular $\mathrm{pH}$ and electrical homeostasis by interacting with transporters and ion channels (11), and is reported to protect the cell membrane from lipid peroxidation by inhibiting the production of malondialdehyde (MDA), which is a result of degradation of polyunsaturated lipids $(12,13)$. The occurrence of free oxygen radicals, such as MDA, is closely connected with the production of inflammatory cytokines such as inducible nitric oxide synthase (iNOS) and its mediators (14). Therefore, our goal was to determine whether propofol affected the NO pathway and the production of ROS, thereby preventing injury to key proteins of the contractile apparatus, such as MLC2.

'Department of Paediatrics, Maastricht University Medical Center, School of Oncology and Developmental Biology, School of Mental Health and Neuroscience, Maastricht, The Netherlands; ${ }^{2}$ Department of Pediatric Cardiology \& Intensive Care Medicine, University of Göttingen, Göttingen, Germany; ${ }^{3}$ Department of Physiology, Cardiovascular Research Institute Maastricht (CARIM) Maastricht University Medical Center, Maastricht, The Netherlands; ${ }^{4}$ Regenerative Medicine Program, Sprott Centre for Stem Cell Research at the Ottawa Hospital Research Institute, Ottawa, Ontario, Canada. Correspondence: Boris W. Kramer (b.kramer@maastrichtuniversity.nl)

Received 24 June 2015; accepted 5 November 2015; advance online publication 10 February 2016. doi:10.1038/pr.2016.10 


\section{RESULTS}

\section{M-mode Echocardiography}

Due to logistical limitations, echocardiography at baseline could only be performed in 24 out of 36 late-preterm lambs, and measurements after $8 \mathrm{~h}$ were limited to 16 out of 36 animals, as previously stated (4). We therefore present the individual measurements per group and compared only the medians of the groups. Measurements are considered as trends.

Echocardiography at baseline. The median baseline fractional shortening (FS) $1 \mathrm{~h}$ after birth was $48.5 \%$ in the propofol group and $46.0 \%$ in the isoflurane control group (Figure 1). The baseline FS in asphyctic lambs was $55.0 \%$ in the propofol group and $57.0 \%$ in the isoflurane group. The baseline FS was higher after resuscitation, and adrenaline treatment in the propofol asphyxia group compared with the propofol control group. A similar difference was observed between the two isoflurane groups.

Echocardiography after $8 \mathrm{~h}$. FS 8-h postnatal and before sacrifice was $57.0 \%$ in the propofol group and $55.5 \%$ in the isoflurane control group. Compared with the baseline values, FS increased in the propofol and the isoflurane-treated animals. The final FS in the asphyctic lambs was $46.0 \%$ in the propofol and $39.5 \%$ in the isoflurane group. In comparison with the baseline values, FS decreased in the propofol and the isoflurane-treated lambs. The FS at the end of the experiment was lower in the propofol asphyxia compared with the propofol control group. A similar difference was observed between the two isoflurane groups.

\section{Lipid Peroxidation Assay}

Malondialdehyde. In the propofol and isoflurane control group, MDA level in cardiac left ventricular tissue remained unchanged compared with the gestational age (GA) control

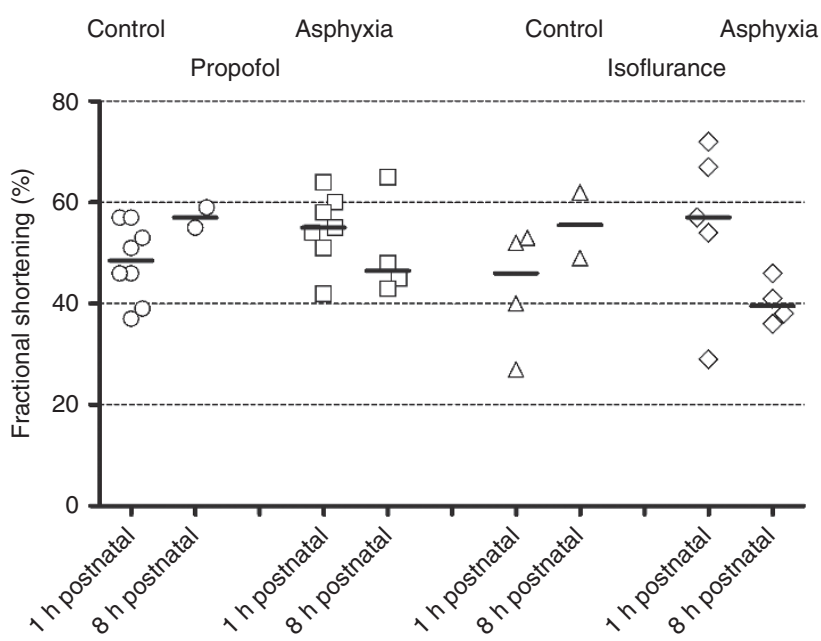

Figure 1. M-mode echocardiography: left ventricular fractional shortening (LVFS) in various groups. Because of technical reasons, LVFS could not be determined in all the animals. However, a trend for a decreasing median could be observed in the isoflurane-treated asphyctic group at the end of the experiment, whereas the decrease in the propofol-treated asphyctic group was less distinct. Circles symbolize the propofol control group, squares symbolize the propofol-treated asphyctic group, triangles symbolize the isoflurane control group, and diamonds symbolize the isoflurane-treated asphyctic group. group (Figure 2). MDA increased by $364.0 \%$ in the propofol asphyxia group compared with the propofol control $(P<0.05)$ and by $330.3 \%(P<0.05)$ compared with the GA control group. In the isoflurane asphyxia group, MDA increased by $730.1 \%(P$ $<0.05)$ compared with the GA control group and to $681.1 \%$ $(P<0.05)$ when compared with the isoflurane control group. MDA was reduced by $54.7 \%(P<0.05)$ in asphyxiated lambs if these lambs underwent treatment with propofol in comparison to treatment with isoflurane.

\section{Western Blot Analysis}

Myosin light chain 2. In the propofol asphyxia group, MLC2 decreased to $51.7 \%$ ( $P=$ NS, Figure 3a) compared with GA and to $57.1 \%(P=\mathrm{NS})$ in comparison with the propofol control group. After prenatal asphyxia, MLC2 decreased in the isoflurane treated lambs to $9.5 \%$ compared with GA controls $(P<0.05)$. Compared with the isoflurane control group, MLC2 decreased to $8.3 \%(P<0.05)$ and to $18.3 \%(P<0.05)$ when compared with the propofol asphyxia group.

Inducible nitric oxide synthase. In the propofol asphyxia group, iNOS increased slightly to $228.7 \%(P=$ NS, Figure $3 \mathbf{b})$ compared with GA and to $208.5 \%(P=\mathrm{NS})$ in comparison to the propofol control group. After prenatal asphyxia, iNOS increased in isoflurane treated lambs to $486.4 \%$ compared with GA controls $(P<0.05)$, to $313.4 \%(P<0.05)$ compared with the isoflurane control group, and to $212.7 \%(P<0.05)$ compared with the propofol asphyxia group.

P38 ( 238 MAPK). In the propofol and isoflurane control group, p38 MAPK level in cardiac left ventricular tissue remained

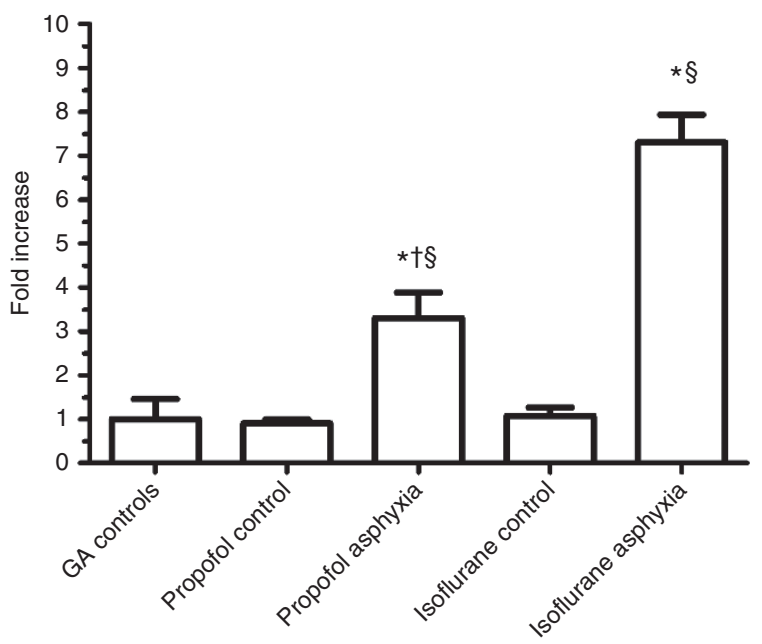

Figure 2. Reactive oxygen species (ROS) in fetal ovine hearts. Malondialdehyde (MDA) levels in left ventricular tissue as indication of lipid peroxidation due to ROS formation. Levels of the gestational age control group were set to 1 . Depicted are mean and SEM. Significant differences $(P<0.05)$ compared with gestational age control groups are marked by *. Significant differences $(P<0.05)$ between asphyxiated and corresponding not-asphyxiated drug control group are marked by $\S$. Significant differences $(P<0.05)$ between the propofol-treated asphyctic group and the isoflurane-treated asphyctic group are marked by †. GA, gestational age. 
a

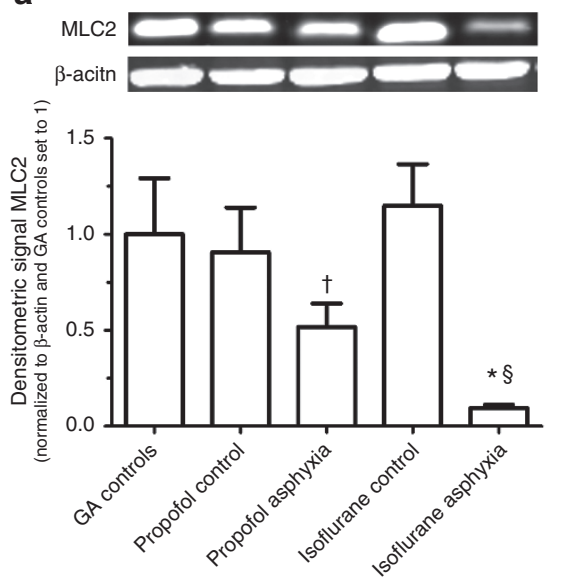

b

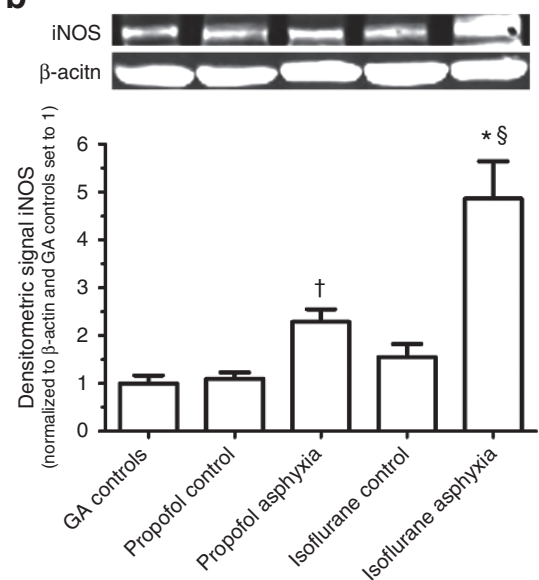

C

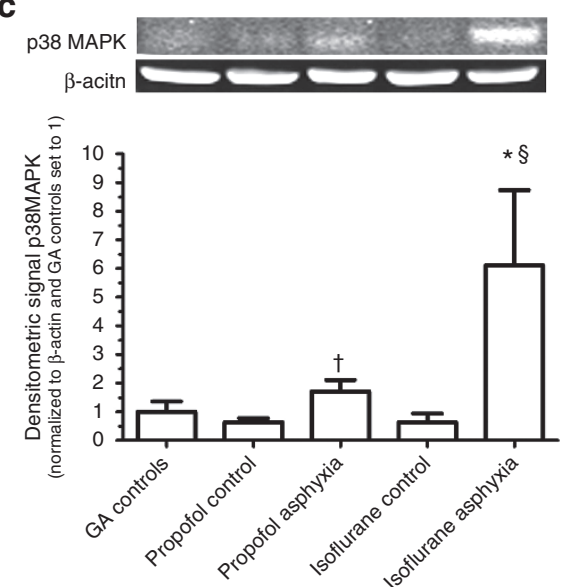

Figure 3. Western blot tests for: (a) myosin light chain 2 (MLC2), (b) inducible nitric oxide synthase (iNOS), and (c) p38 mitogen-activated protein kinase. The corresponding levels in the gestational age (GA) control group were set at 1. The data are depicted as mean values and SEMs. Significant differences $(P<0.05)$ as compared with GA control groups $(n=8)$ are marked by *. Significant differences $(P<0.05)$ between asphyctic (propofol-treated $n=10 ;$ isoflurane-treated $n=8$ ) and the corresponding asphyxia-free control group (propofol-treated $n=11$; isoflurane-treated $n=7$ ) are marked by $\S$. Significant differences $(P<0.05)$ between the propofol-treated asphyctic group and the isoflurane-treated asphyctic group are marked by + .

unchanged compared with the GA control group (Figure 3c). After prenatal asphyxia, p38 MAPK increased in isofluranetreated lambs to $610.6 \%$ compared with GA controls $(P<$ $0.05)$, to $972.3 \%(P<0.05)$ compared with the isoflurane control group, and to $357.7 \%(P<0.05)$ compared with the propofol asphyxia group.

\section{DISCUSSION}

This is the first study to demonstrate that perinatal propofol administration to a severely asphyxiated near-term lamb results in reduced production of ROS and less activation of the NO pathway when compared with an isoflurane regime. As overproduction of ROS and NO is harmful for the contractile apparatus of the heart, this treatment might be beneficial for the heart function of severely asphyxiated newborns.

$\mathrm{NO}$ is a vasoactive gas which is a crucial regulator of cardiac contractility (15). The concentration of endogenous NO under physiological conditions is regulated mostly by neuronal NO-synthase and endothelial NO-synthase. In an inflammatory condition, such as sepsis or hypoxia, the NO level is mainly produced by the inducible NO-synthase (iNOS) $(16,17)$. Therefore, we used iNOS levels in left ventricular tissue to estimate activation of the NO pathway during ischemiareperfusion injury. The myocardial iNOS expression after asphyxia was significantly lower in lambs treated with propofol compared with isoflurane. As previously published (4), the propofol-treated lambs showed significantly lower troponin $\mathrm{T}$ and a slightly better cardiac function, which may indicate that less iNOS expression may be beneficial.

In the literature, the findings in regard to the role of $\mathrm{NO}$ in ischemia-reperfusion injury are inconclusive. Some studies have claimed that both endogenous and exogenous NO can prevent the heart from ischemia-reperfusion injury, whereas other studies have shown that NO increased the myocardial damage during reperfusion (18-20). A possible explanation for the different findings may come from a study by Rakhit et al. (21) who found indications that NO becomes a proapoptotic factor when it reacts with ROS such as superoxide anion to form peroxynitrite.

ROS is generated during myocardial ischemia and reperfusion; it assaults intracellular organelles, cell membranes, and biological macromolecules, including nucleic acid, protein, and lipid, resulting in oxidative stress and cell apoptosis $(22,23)$. We found that the level of ROS was increased in both asphyxia groups compared with controls in our model. In conjunction with the findings of Rakhit et al. (21), our results suggest that the iNOS activation in this setting has more detrimental than beneficial effects.

Several studies have shown that ROS and NO interact to form peroxynitrite, affecting not only the apoptotic pathway but also proteins of the contractile apparatus of the heart, leading to systolic dysfunction $(9,24,25)$. One key target is MLC2 which plays an essential role in the interplay between myosin and actin, which are responsible for the ability of the heart muscle to contract (9). Animal studies in both adults and neonates have shown that oxidative stress due to hypoxiareoxygenation led to MLC2 modifications and its subsequent degradation resulting in severe systolic dysfunction $(9,26)$. In our model, the protein level of MLC2 decreased after severe asphyxia only in the isoflurane-treated lambs. Propofol, in contrast, prevented MLC2 from being degraded, resulting in less cardiac dysfunction.

In addition, the increased cytoskeleton damage in the isoflurane-treated hypoxic hearts in comparison to the propofol exposed hearts is, in our asphyxia model, expressed by the upregulation of mitogen-activated protein kinase (MAPK) p38. The p38/MAPK pathway is activated under hypoxic conditions when the microtubular structure breaks down (27). It has been shown that p38 MAPK is activated as a survival pathway to increase calcium sensitivity and to induce the phosphorylation 
of MLC2 (28). The same effect could be demonstrated with the administration of propofol (10). But this survival mechanism seems to be counteracted if high NO levels are present due to iNOS activation. In this case, the activation of p38 MAPK resulted in cell death $(29,30)$.

Cardiopulmonary resuscitation is a cornerstone of neonatal critical care practice. Severe asphyxia followed by a systemic inflammatory response syndrome often causes left ventricular dysfunction leading to cardiogenic shock and the development of multiorgan failure (7). The lack of oxygen initially triggers an inflammatory response, leading to a massive ROS production which is detrimental for the outcome of the newborn (31). In our study, we were able to show that propofol is effective in an animal model of preterm lambs to reduce the effect of ROS. The underlying reason is likely its biochemical structure, allowing propofol to scavenge and inactivate ROS (11). This corresponds to data deriving from clinical trials in adult medicine. In these trials, it was shown that propofol is effective in patients with left ventricular dysfunction caused by sepsis or myocardial infarction $(32,33)$. The occurrence of ROS, however, is not the only fatal step towards cardiogenic shock and multiorgan failure. In the past two decades it has become apparent that the upregulation of iNOS transcription and overproduction of NO causes catecholamine-resistant vasodilatation and reduced myocardial inotropy, resulting in hypotension and a fall in cardiac output (34). NO can also react with ROS to produce peroxynitrate, a molecule directly toxic to the cells via modification of proteins and DNA (34). In our model, propofol decreases the iNOS activation and thereby the damage of the contractile apparatus, preventing key structures, such as MLC2, from nitration and degradation. In the literature, it has been shown that propofol is not only a direct scavenger of ROS, but also of peroxynitrate. Additionally, propofol reduces the activation of iNOS by interfering with the AKT/IL-6/signal transducer and activator of transcription-3 pathway $(4,5,11,35)$. Unfortunately, identifying and blocking molecular key steps like the activation of iNOS in the pathophysiology of severe ischemia with resulting deterioration of left ventricular function, cardiogenic shock, and multiorgan failure does not necessarily lead to an improved patient outcome as we learned from the TRIUMPH trial (34). Despite promising data from animal experiments, the use of nonselective NOS inhibitors did not result in an improved outcome, but even more harm in treated patients, which led to a premature termination of this trial. Therefore, it is important to identify therapeutic options which specifically target the right steps in the pathophysiological cascade. Propofol seems to be a very promising agent in that context $(11,33,36)$, but still has to prove its effectiveness in the human neonatal population. One major concern in this respect is propofol infusion syndrome. Though rare, it is much more likely to occur in the pediatric population than the adult population. It leads to bradyarrythmias and often a therapy-refractory arterial hypotension (37). We showed earlier that there was no indication for propofol infusion syndrome with our treatment regime in our animal cohort (4), but as mentioned above, it is rare and will probably only be seen if propofol is used in larger patient cohorts. To prevent pediatric patients from developing a propofol infusion syndrome, it is recommended to not exceed a dosage of more than $4 \mathrm{mg} / \mathrm{kg} / \mathrm{h}$ for more than $48 \mathrm{~h}$ (37). We used a continuous propofol infusion of not more than $3 \mathrm{mg} / \mathrm{kg} / \mathrm{h}$ for $8 \mathrm{~h}$ and could still observe beneficial effects of propofol on the lambs' outcome.

However, there are additional limitations to our study. First of all, due to logistical limitations, we could not perform echocardiography in all animals at all planned time points. Therefore, we displayed the echocardiography data as scatter plot and interpreted the differences in means only as a trend without doing a proper statistical analysis.

In addition, our study design allows only a general correlation of molecular and functional data. A systemic model has the advantage of displaying clinically relevant pathways; however, a more appropriate approach, such as a cell culture model, would be necessary to determine the degree to which a particular molecular pathway contributed to a defined functional outcome.

In this study, we were able to highlight some key ways in which a drug such as propofol with its multiple complex properties interacts on a cellular level in an in vivo model of severe perinatal asphyxia. Further research is needed to understand in detail how the beneficial effects are mediated and if there is a possibility to develop new drugs that make more successful use of the identified targets.

\section{Conclusion}

Each year, perinatal asphyxia contributes to over one million newborn deaths worldwide. Resuscitation is crucial to restore oxygenation to deprived organs, including the heart. However, reoxygenation of asphyxiated newborns may lead to generation of ROS and NO, causing further myocardial damage by nitration of key proteins of the contractile apparatus. In this animal study, we could demonstrate that pre- and postresuscitation administration of propofol to the maternal fetal unit reduced the myocardial oxidative stress and caused an improvement in cardiac function in late-preterm lambs suffering from severe asphyxia and cardiac arrest.

\section{MATERIAL AND METHODS \\ Animals}

The study was approved by the Animal Ethics Research Committee, Maastricht University, The Netherlands. As previously described (3-5), Texel ewes were date-mated and the fetuses were randomized for umbilical cord occlusion and sedation. Experiments were made on 44 late-preterm fetal sheep of both genders at GA of 133 or 134 days (term $150 \mathrm{~d}$ ). Eighteen sheep fetuses were subjected to total umbilical cord occlusion in utero, and 18 fetal sheep served as sham controls. In each group, half of the pregnant ewes and their offspring were sedated with propofol and the other half with isoflurane. Eight lambs served as GA controls to obtain the reference values for the molecular pathways in this age group. To avoid any influence by anaesthetic drugs in this group, the pregnant ewes were euthanized by an injection of T61 (Veterinaria AG, Zürich, Switzerland) at gestational day 133 or 134 and their offspring were delivered surgically immediately afterwards and directly euthanized by an injection of T61 (Veterinaria AG).

\section{Experimental Protocol}

The pregnant ewes of the isoflurane group were intubated after thiopenthal induction $(15 \mathrm{mg} / \mathrm{kg})$, and general anaesthesia was maintained with isoflurane $(1-2 \%)$. The pregnant ewes of the propofol group 
received propofol both as induction bolus $(6 \mathrm{mg} / \mathrm{kg})$ and for general anaesthesia $(25 \mathrm{mg} / \mathrm{kg} / \mathrm{h})$ during the cesarean delivery. Both anaesthesia types were supplemented by continuous remifentanyl infusion ( $3 \mu \mathrm{g} / \mathrm{kg} / \mathrm{min})$. After a lower midline incision, the fetus was carefully extracted through a small incision of the uterus. An endotracheal tube was inserted and subsequently clamped to prevent fetal breathing. Catheters were placed in the femoral and umbilical artery and in the jugular vein and used for baseline blood sampling (Abbott i-signal transducer and activator of transcription 1 Blood Gas Analyzer, Abbott Laboratories, IL) and for continuous monitoring of fetal mean arterial blood pressure and heart rate. Electrocardiogram and electroencephalogram electrodes were placed and stitched to the skin. The umbilical cord was gently extracted through the incision of the uterus and a vascular occluder (OC16HD, 16mm, In Vivo Metric, Healdsburg, CA) was placed around the umbilical cord. The aim of the occlusion was a mean arterial blood pressure below $30 \mathrm{mmHg}$ for $2 \mathrm{~min}$. At the end of the occlusion, the lambs always suffered from severe bradycardia (heart rate $<30 / \mathrm{min}$ ) or even complete cardiac arrest. If there was no increase in heart rate after $20 \mathrm{~s}$ after the end of occlusion, resuscitation was started. The resuscitation protocol included immediate start of cardiac massage with a frequency of $120 / \mathrm{min}$ and adrenaline administration in augmenting dosage from 30 over $60 \mu \mathrm{g}$ to $0.1 \mathrm{mg}$. In addition, a volume bolus of Ringers lactate of $10 \mathrm{ml} / \mathrm{kg} \mathrm{BW}$ was administered after the first shot of adrenaline and ventilation was given with a balloon $\left(\mathrm{FiO}_{2}=0.21\right.$, frequency $\left.=60 / \mathrm{min}\right)$. After its umbilical cord was cut, the fetus was brought to an open, heated incubator (IW930 Series CosyCot Infant Warmer, Fisher \& Paykel Healthcare, Auckland, New Zealand), maintaining a body temperature of $38.5^{\circ} \mathrm{C}$ and resuscitation was continued. The lambs were connected to pressure-regulated ventilation using a ventilator Servo 900C (Siemens-Elema, Solna, Sweden) with initial settings as follows: $\mathrm{FiO}_{2}=1$, PEEP $5 \mathrm{cmH}_{2} \mathrm{O}$, PiP $30 \mathrm{cmH}_{2} \mathrm{O}$, frequency $60 / \mathrm{min}$, I:E 1:2. Thereafter, inspiratory pressure was adjusted to achieve a targeted minute volume of $3.0 \mathrm{l} / \mathrm{min}$ and a $\mathrm{PaCO}_{2}$ of $35-45$ $\mathrm{mmHg}$. The ventilation and sedation was continued for $8 \mathrm{~h}$ after delivery. The sedation was maintained with the same anesthetic which was used for maternal sedation (either with isoflurane $(0.5-1.0 \%)$ or propofol $(1-3 \mathrm{mg} / \mathrm{kg} / \mathrm{h}))$ and was supplemented with remifentanyl $(3 \mu \mathrm{g} /$ $\mathrm{kg} / \mathrm{min}$ ) in both groups. The remifentanyl was dissolved in $20 \%$ glucose and Ringers lactate solution in a concentration allowing for an infusion rate of $3 \mathrm{~mL} / \mathrm{kg} / \mathrm{h}$. All lambs (sham treated included) received surfactant $(200 \mathrm{mg} / \mathrm{kg}$ BW Curosurf, Chiesi, Parma, Italy) intratracheally and two maternal blood transfusions of $10 \mathrm{ml} / \mathrm{kg}$ BW during initial stabilization. All cord-occluded lambs developed a spontaneous heart rate $(>150 / \mathrm{min})$ and a sufficient mean arterial blood pressure $(>50 \mathrm{mmHg}$ ) within $10 \mathrm{~min}$ of starting resuscitation with the exception of two lambs, which were excluded from the study.

The 18 sham-treated fetuses were prepared in utero, with catheters inserted in the femoral and umbilical artery and jugular vein and endotracheal intubation as described above. Eleven minutes (mean time of occlusion in study group) after the end of the instrumentation, they were delivered, weighed, sedated, and connected to pressurecontrolled ventilation (see above).

At $8 \mathrm{~h}$ after delivery, the lambs were euthanized by an intravenous injection of T61 (Veterinaria AG). The heart was immediately taken and shock frozen in liquid nitrogen. Tissue was subsequently stored at $-80^{\circ} \mathrm{C}$.

\section{M-mode Echocardiography}

A transthoracic echocardiography using a $5.2-\mathrm{MHz}$ transducer (Megas CVX, Esaote, Genova, Italy) was performed at baseline $(1 \mathrm{~h}$ postnatally) and prior to sacrifice ( $8 \mathrm{~h}$ after birth). M-mode recordings from a parasternal short axis view were used to determine FS according to the American Society of Echocardiography recommendations (38). The investigator performing and reading the echocardiogram was blinded to the treatment allocation.

\section{Lipid Peroxidation Assay}

Lipid peroxides include reactive aldehydes, of which the most abundant is MDA. Therefore, measurement of MDA is widely used as an indicator of lipid peroxidation (39). For heart tissue analysis, we used the ALdetect (MDA-Specific) Lipid Peroxidation Assay Kit (ALdetect (MDA-Specific) Lipid Peroxidation Assay Kit BML-AK-171, Enzo
Life Sciences International, Plymouth Meeting, PA) according to the manufacture's manual.

\section{Western Blotting}

Frozen left ventricular heart tissue was homogenized in ice-cold RIPA buffer (Sigma-Aldrich, St. Louis, MO) containing Halt Protease and Phosphatase Inhibitor Cocktail, EDTA-free (100x) (Thermo Fisher Scientific, Rockford, IL). The samples were then centrifuged at $500 \times g$ for $20 \mathrm{~min}$ at $4{ }^{\circ} \mathrm{C}$ to remove cellular debris. Protein content in the supernatant was determined using the Micro BCA Protein Assay Kit (Thermo Fisher Scientific, Rockford, IL), with BSA as the standard. Western blots were performed as previously described (40). The following dilutions for antibodies were used: 1:1,000 for monoclonal anti- $\beta$-actin clone AC-1 (Sigma-Aldrich), anti-MLC antibody (Abcam, Cambridge, UK), anti-iNOS (BD Transduction Laboratories, Franklin Lakes, NJ), anti-TAK-1 (Cell Signaling Technology, Danvers, MA), and anti-phospho-p38 MAPK out of the PathScan Multiplex Western Cocktail II (Cell Signaling Technology). As secondary antibody IRDye 680-conjugated goat (polyclonal) antirabbit IgG, IRDye 800-conjugated goat (polyclonal) antirabbit IgG, IRDye 680-conjugated goat (polyclonal) antimouse IgG, and IRDye 800-conjugated goat (polyclonal) antimouse IgG, (LI-COR, Lincoln, Nebraska) were used in a dilution of 1:10,000. The blots were analyzed using the LICOR Odyssey Infrared Imaging System and the signal of the target proteins was normalized to the signal of $\beta$-actin which acts as a house-keeping protein. Images were acquired using Adobe Photoshop CS6 software (Adobe Systems Incorporated, San Jose, CA).

\section{Statistical Analysis}

Measurements of the left ventricular fractional shortening were depicted as median. All other data were depicted as mean \pm SEM. Statistical significance between different groups was analyzed by the analysis of variance (ANOVA) followed by the Student-NewmanKeuls multiple comparison test. Differences in $P$ value $<0.05$ were considered significant. All statistical analyses were performed using the statistical software GraphPad Prism 5.0 (La Jolla, CA).

\section{ACKNOWLEDGMENTS}

We thank Monique Engel, MD, for her advice and technical equipment and Reint K. Jellema, MD, Elke Kuypers, MSc, Lotte van den Heuij, BSc, and the staff of the central animal facility of Maastricht University for their excellent technical help.

\section{STATEMENT OF FINANCIAL SUPPORT}

This work was supported by the Foundation of Pediatrics (Stichting Kindergeneeskunde), Maastricht University Medical Center.

Disclosure: The authors declared no conflict of interest.

\section{REFERENCES}

1. Szymankiewicz M, Matuszczak-Wleklak M, Vidyasagar D, Gadzinowski J. Retrospective diagnosis of hypoxic myocardial injury in premature newborns. J Perinat Med 2006;34:220-5.

2. Shah P, Riphagen S, Beyene J, Perlman M. Multiorgan dysfunction in infants with post-asphyxial hypoxic-ischaemic encephalopathy. Arch Dis Child Fetal Neonatal Ed 2004;89:F152-5.

3. Smit AL, Seehase M, Stokroos RJ, et al. Functional impairment of the auditory pathway after perinatal asphyxia and the short-term effect of perinatal propofol anesthesia in lambs. Pediatr Res 2013;74:34-8.

4. Seehase M, Houthuizen P, Jellema RK, et al. Propofol administration to the fetal-maternal unit reduces cardiac injury in late-preterm lambs subjected to severe prenatal asphyxia and cardiac arrest. Pediatr Res 2013;73:427-34.

5. Seehase M, Jennekens W, Zwanenburg A, et al. Propofol administration to the maternal-fetal unit improved fetal EEG and influenced cerebral apoptotic pathway in preterm lambs suffering from severe asphyxia. Mol Cell Pediatr 2015;2:4.

6. Hack M, Stork E. Resuscitation at birth and long-term follow-up. Lancet 2009;373:1581-2.

7. Lawn JE, Cousens S, Zupan J; Lancet Neonatal Survival Steering Team. 4 million neonatal deaths: when? where? why? Lancet 2005;365:891-900. 
8. Thomas SA, Fallavollita JA, Lee TC, Feng J, Canty JM Jr. Absence of troponin I degradation or altered sarcoplasmic reticulum uptake protein expression after reversible ischemia in swine. Circ Res 1999;85:446-56.

9. Doroszko A, Polewicz D, Cadete VJ, et al. Neonatal asphyxia induces the nitration of cardiac myosin light chain 2 that is associated with cardiac systolic dysfunction. Shock 2010;34:592-600.

10. Kanaya N, Gable B, Murray PA, Damron DS. Propofol increases phosphorylation of troponin I and myosin light chain 2 via protein kinase $\mathrm{C}$ activation in cardiomyocytes. Anesthesiology 2003;98:1363-71.

11. Vasileiou I, Xanthos T, Koudouna E, et al. Propofol: a review of its nonanaesthetic effects. Eur J Pharmacol 2009;605:1-8.

12. Sanders RD, Hussell T, Maze M. Sedation \& immunomodulation. Crit Care Clin 2009;25:551-70, ix.

13. Kokita N, Hara A. Propofol attenuates hydrogen peroxide-induced mechanical and metabolic derangements in the isolated rat heart. Anesthesiology 1996;84:117-27.

14. Umar S, van der Laarse A. Nitric oxide and nitric oxide synthase isoforms in the normal, hypertrophic, and failing heart. Mol Cell Biochem 2010;333:191-201.

15. Ziolo MT, Bers DM. The real estate of NOS signaling: location, location, location. Circ Res 2003;92:1279-81.

16. Massion PB, Balligand JL. Modulation of cardiac contraction, relaxation and rate by the endothelial nitric oxide synthase (eNOS): lessons from genetically modified mice. J Physiol 2003;546:63-75.

17. Bruckdorfer R. The basics about nitric oxide. Mol Aspects Med 2005;26:3-31.

18. Williams MW, Taft CS, Ramnauth S, Zhao ZQ, Vinten-Johansen J. Endogenous nitric oxide (NO) protects against ischaemia-reperfusion injury in the rabbit. Cardiovasc Res 1995;30:79-86.

19. Woolfson RG, Patel VC, Neild GH, Yellon DM. Inhibition of nitric oxide synthesis reduces infarct size by an adenosine-dependent mechanism. Circulation 1995;91:1545-51.

20. Weerateerangkul P, Chattipakorn S, Chattipakorn N. Roles of the nitric oxide signaling pathway in cardiac ischemic preconditioning against myocardial ischemia-reperfusion injury. Med Sci Monit 2011;17:RA44-52.

21. Rakhit RD, Marber MS. Nitric oxide: an emerging role in cardioprotection? Heart 2001;86:368-72.

22. Dröge W. Free radicals in the physiological control of cell function. Physiol Rev 2002;82:47-95.

23. Ahn HJ, Kim KI, Kim G, Moon E, Yang SS, Lee JS. Atmospheric-pressure plasma jet induces apoptosis involving mitochondria via generation of free radicals. PLoS One 2011;6:e28154.

24. White MY, Cordwell SJ, McCarron HC, Tchen AS, Hambly BD, Jeremy RW. Modifications of myosin-regulatory light chain correlate with function of stunned myocardium. J Mol Cell Cardiol 2003;35:833-40.
25. Ferdinandy P, Schulz R. Nitric oxide, superoxide, and peroxynitrite in myocardial ischaemia-reperfusion injury and preconditioning. Br J Pharmacol 2003;138:532-43.

26. Akiyama K, Akopian G, Jinadasa P, et al. Myocardial infarction and regulatory myosin light chain. J Mol Cell Cardiol 1997;29:2641-52.

27. Teng M, Jiang XP, Zhang Q, et al. Microtubular stability affects pVHLmediated regulation of HIF-1alpha via the p38/MAPK pathway in hypoxic cardiomyocytes. PLoS One 2012;7:e35017.

28. Yang G, Li T, Xu J, Peng X, Liu L. Mitogen-activated protein kinases regulate vascular reactivity after hemorrhagic shock through myosin light chain phosphorylation pathway. J Trauma Acute Care Surg 2013;74: 1033-43.

29. Brown GC. Nitric oxide and neuronal death. Nitric Oxide 2010;23:153-65.

30. Monsuez JJ, Escaut L, Teicher E, Charniot JC, Vittecoq D. Cytokines in HIV-associated cardiomyopathy. Int J Cardiol 2007;:150-7.

31. Mondal N, Bhat BV, Banupriya C, Koner BC. Oxidative stress in perinatal asphyxia in relation to outcome. Indian J Pediatr 2010;77:515-7.

32. Bovill JG. Intravenous anesthesia for the patient with left ventricular dysfunction. Semin Cardiothorac Vasc Anesth 2006;10:43-8.

33. Darrouj J, Karma L, Arora R. Cardiovascular manifestations of sedatives and analgesics in the critical care unit. Am J Ther 2009;16:339-53.

34. Bailey A, Pope TW, Moore SA, Campbell CL. The tragedy of TRIUMPH for nitric oxide synthesis inhibition in cardiogenic shock: where do we go from here? Am J Cardiovasc Drugs 2007;7:337-45.

35. Hsing CH, Lin MC, Choi PC, et al. Anesthetic propofol reduces endotoxic inflammation by inhibiting reactive oxygen species-regulated Akt/IKK $\beta$ / NF- $\kappa B$ signaling. PLoS One 2011;6:e17598.

36. Yildirim V, Doganci S, Aydin A, Bolcal C, Demirkilic U, Cosar A. Cardioprotective effects of sevoflurane, isoflurane, and propofol in coronary surgery patients: a randomized controlled study. Heart Surg Forum 2009;12:E1-9.

37. Wong JM. Propofol infusion syndrome. Am J Ther 2010;17:487-91.

38. Lai WW, Geva T, Shirali GS, et al.; Task Force of the Pediatric Council of the American Society of Echocardiography; Pediatric Council of the American Society of Echocardiography. Guidelines and standards for performance of a pediatric echocardiogram: a report from the Task Force of the Pediatric Council of the American Society of Echocardiography. J Am Soc Echocardiogr 2006;19:1413-30.

39. Esterbauer H, Schaur RJ, Zollner H. Chemistry and biochemistry of 4-hydroxynonenal, malonaldehyde and related aldehydes. Free Radic Biol Med 1991;11:81-128.

40. Seehase M, Gantert M, Ladenburger A, et al. Myocardial response in preterm fetal sheep exposed to systemic endotoxinaemia. Pediatr Res 2011;70:242-6. 